\title{
Two New Aspidosperma Indole Alkaloids from Yunnan Kopsia arborea
}

\author{
Yuqiu Wu, ${ }^{a}$ Mariko Kitajima, ${ }^{a}$ Noriyuki Kogure, ${ }^{a}$ Yunsong Wang, ${ }^{b}$ Rongping Zhang, ${ }^{c}$ and \\ Hiromitsu TAKAYAMA $*, a$ \\ ${ }^{a}$ Graduate School of Pharmaceutical Sciences, Chiba University; 1-33 Yayoi-cho, Inage-ku, Chiba 263-8522, Japan: \\ ${ }^{b}$ Key Laboratory of Medicinal Chemistry for Natural Resources, Ministry of Education, Research School of Pharmacy, \\ Yunnan University; Kunming 650031, Yunnan Province, China: and ${ }^{c}$ Department of Pharmaceutical Sciences, Kunming \\ Medical University; Kunming 650031, Yunnan Province, China.
}

Received January 9, 2010; accepted April 14, 2010; published online April 15, 2010

Two new indole alkaloids, kopsiyunnanines G (1) and H (2), possessing the Aspidosperma skeleton were isolated from the aerial part of Yunnan Kopsia arborea BLume (Apocynaceae). Their structures and stereochemistry were elucidated by means of MS and 2D NMR analyses.

Key words Apocynaceae; Kopsia; indole alkaloid; structure elucidation; NMR

Kopsia plants are widely distributed from South China and Burma to northern Australia and Vanuatu ${ }^{1)}$ and are noted for producing a great variety of monoterpenoid indole alkaloids. Phytochemical work on various Kopsia plants, particularly those indigenous to Malaysia, has led to the isolation of more than 180 alkaloids. ${ }^{2)}$ The alkaloids often possess unusual skeletons and useful bioactivities, ${ }^{3-8)}$ and many of them are intriguing targets of total synthesis. ${ }^{9-11)}$ As part of our ongoing search for bioactive novel indole alkaloids from various botanical resources ${ }^{12-18)}$ we have reported the isolation of a series of novel alkaloids kopsiyunnanines $\mathrm{A}-\mathrm{F}$ from Yunnan Kopsia arborea Blume (Apocynaceae). ${ }^{19-21)}$ We describe herein the structure elucidation of two new minor compounds having an Aspidosperma skeleton (Fig. 1).

\section{Results and Discussion}

The $\mathrm{MeOH}$ extract of the aerial part of Yunnan K. arborea was subjected to a conventional procedure ${ }^{19-21)}$ to give the crude base, which was roughly separated by $\mathrm{SiO}_{2}$ column chromatography into 9 fractions using $\mathrm{CHCl}_{3}-\mathrm{MeOH}$ gradient as eluent. The $10-40 \% \mathrm{MeOH} / \mathrm{CHCl}_{3}$ fractions from the first column chromatography were subjected to repeated column chromatography to give two new indole alkaloids, kopsiyunnanine $\mathrm{G}(\mathbf{1})$ and kopsiyunnanine $\mathrm{H}(\mathbf{2})$, together with 9 known compounds: eburenine $(3),{ }^{22)}(-)$-eburnamine, ${ }^{23)}(-)$ methyleburnamine, ${ }^{24)}$ (-)-ethyleburnamine, ${ }^{25)}(+)$-isoebur-

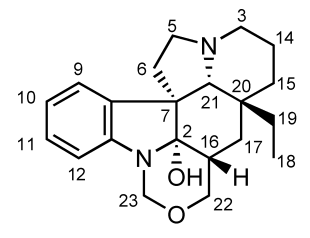

Kopsiyunnanine G (1)

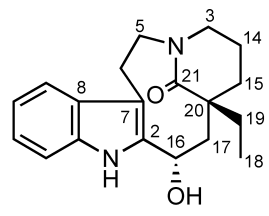

Kopsiyunnanine H (2)

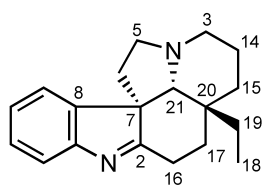

Eburenine (3) namine ${ }^{23)}(+)$-methylisoeburnamine ${ }^{24)}(+)$-ethylisoeburnamine, ${ }^{26)}(+)$-eburnamenine, ${ }^{27)}$ and $(+)$-eburnamonine. ${ }^{28)}$ The identification of known compounds was done by analyzing spectroscopic data and comparing their physicochemical data with those published in the literature.

Compound 1, named kopsiyunnanine G, was obtained as a colorless amorphous solid. The UV spectrum exhibited absorption maxima at $\lambda_{\max } 291.5,246.0$, and $208.0 \mathrm{~nm}$, suggesting a typical indoline chromophore. It displayed a molecular ion peak at $m / z 340.2157\left[\mathrm{M}^{+}\right]$(Calcd 340.2151) in the HR-electron ionization-mass spectra (EI-MS) spectrum, which corresponded to the molecular formula $\mathrm{C}_{21} \mathrm{H}_{28} \mathrm{~N}_{2} \mathrm{O}_{2}$ requiring 9 degrees of unsaturation. The ${ }^{1} \mathrm{H}-\mathrm{NMR}$ spectrum of 1 (Table 1) showed signals assignable to four aromatic protons $\left(\delta_{\mathrm{H}} 7.09\right.$, dd, H-9; $\delta_{\mathrm{H}} 6.79$, ddd, H-10; $\delta_{\mathrm{H}} 7.12$, ddd, $\mathrm{H}-11 ; \delta_{\mathrm{H}} 6.71$, brd, $\left.\mathrm{H}-12\right)$ in the benzene ring and an ethyl side chain (judging from the triplet signal for the methyl function $\delta_{\mathrm{H}} 0.71$ with $J=7.5 \mathrm{~Hz}$ ). ${ }^{13} \mathrm{C}-\mathrm{NMR}$ (Table 1) and distortionless enhancement by polarization transfer (DEPT) spectra demonstrated a total of 21 carbons, including $2 s p^{2}$ quaternary carbons, $4 s p^{2}$ methine groups, $3 s p^{3}$ quaternary carbons, $2 s p^{3}$ methines, $9 s p^{3}$ methylene groups, and 1 methyl group, in agreement with the molecular formula.

To establish the connections among the above-described structural units, heteronuclear multiple bond connectivity (HMBC) and ${ }^{1} \mathrm{H}-{ }^{1} \mathrm{H}$ correlation spectroscopy (COSY) analyses were carried out. ${ }^{1} \mathrm{H}-{ }^{1} \mathrm{H}$ COSY data enabled the determination of several sets of connections as indicated by bold bonds in Fig. 2. Furthermore, the fragment $\mathrm{C}(21)-\mathrm{N}(4)$ $\mathrm{C}(3)-\mathrm{C}(14)-\mathrm{C}(15)$ was defined through a ${ }^{3} J$ long-range $\mathrm{HMBC}$ correlation from $\mathrm{H}-21$ to $\mathrm{C}-3$ and $\mathrm{C}-15$. Another fragment $\mathrm{C}(21)-\mathrm{N}(4)-\mathrm{C}(5)-\mathrm{C}(6)-\mathrm{C}(7)$ was suggested by the correlations of H-21/C-5 and H-5/C-7. The chemical shifts of the two proton signals resonating unusually downfield $\left(\delta_{\mathrm{H}}\right.$ $5.08,4.87$ with geminal coupling constant of $J=11.0 \mathrm{~Hz}$ ) were assigned to one $s p^{3}$ methylene group $\left(\delta_{\mathrm{C}} 72.6\right)$ that was adjacent to both nitrogen and oxygen atoms. This was confirmed by the $\mathrm{HMBC}$ correlations from $\mathrm{H}-23$ to $\mathrm{C}-2$ and $\mathrm{C}-$ 13. The chemical shifts of two other protons resonating downfield at $\delta_{\mathrm{H}} 3.91$ and $\delta_{\mathrm{H}} 3.48$ with a geminal coupling constant of $J=11.0 \mathrm{~Hz}$ indicated the existence of an oxygenated $s p^{3}$ methylene group $\left(\delta_{\mathrm{C}} 67.8\right)$. This group associated with the same oxygen atom as the $\mathrm{C}-23$ methylene

Fig. 1. Structures of Compounds $\mathbf{1}-\mathbf{3}$ 
Table 1. ${ }^{1} \mathrm{H}-\mathrm{NMR}(J$ in $\mathrm{Hz})$ and ${ }^{13} \mathrm{C}-\mathrm{NMR}$ Data for $\mathbf{1}$ and $\mathbf{2}$ in $\mathrm{CDCl}_{3}$

\begin{tabular}{|c|c|c|c|c|}
\hline \multirow{2}{*}{ Position } & \multicolumn{2}{|l|}{1} & \multicolumn{2}{|l|}{2} \\
\hline & $\delta_{\mathrm{H}}(\mathrm{mult}, \mathrm{Hz})^{a)}$ & $\delta_{\mathrm{C}}^{b)}$ & $\delta_{\mathrm{H}}(\text { mult }, \mathrm{Hz})^{c)}$ & $\delta_{\mathrm{C}}^{b)}$ \\
\hline $\mathrm{NH}$ & & & 8.38 (br s) & \\
\hline 2 & & 93.0 & & 138.0 \\
\hline \multirow[t]{2}{*}{3} & $3.06(\mathrm{~m})$ & 53.4 & $2.78(\mathrm{~m})$ & 52.0 \\
\hline & $1.97(\mathrm{ddd}, 13.5,11.0,3.0)$ & & 1.78 (overlapped) & \\
\hline \multirow[t]{2}{*}{5} & 3.04 (ddd, $13.5,9.5,3.5)$ & 52.0 & $4.20(\mathrm{dd}, 13.0,7.0)$ & 47.2 \\
\hline & $2.23(\mathrm{~m})$ & & $2.61(\mathrm{ddd}, 13.0,12.0,6.4)$ & \\
\hline \multirow[t]{2}{*}{6} & $2.72(\mathrm{ddd}, 13.5,9.5,7.0)$ & 30.3 & $3.48(\mathrm{ddd}, 13.8,12.0,7.0)$ & 19.7 \\
\hline & $1.29(\mathrm{ddd}, 13.5,11.0,3.5)$ & & $2.88(\mathrm{dd}, 13.8,6.4)$ & \\
\hline 7 & & 55.5 & & 108.0 \\
\hline 8 & & 135.1 & & 128.4 \\
\hline 9 & $7.09(\mathrm{dd}, 7.5,1.0)$ & 123.3 & $7.56(\mathrm{~d}, 7.6)$ & 118.0 \\
\hline 10 & $6.79(\mathrm{ddd}, 7.5,7.5,1.0)$ & 120.0 & $7.12(\mathrm{ddd}, 7.6,7.6,1.2)$ & 119.6 \\
\hline 11 & $7.12(\mathrm{ddd}, 7.5,7.5,1.0)$ & 127.7 & $7.19(\mathrm{ddd}, 7.6,7.6,1.2)$ & 122.2 \\
\hline 12 & $6.71(\mathrm{brd}, 7.5)$ & 109.4 & $7.36(\mathrm{~d}, 7.6)$ & 111.2 \\
\hline 13 & & 147.3 & & 135.4 \\
\hline \multirow[t]{2}{*}{14} & $1.72(\mathrm{~m})$ & 21.5 & $1.28(\mathrm{~m})$ & 19.6 \\
\hline & $1.50(\mathrm{~m})$ & & $1.05(\mathrm{~m})$ & \\
\hline \multirow[t]{2}{*}{15} & 1.64 (overlapped) & 34.2 & $1.64(\mathrm{~m})$ & 31.3 \\
\hline & 1.06 (overlapped) & & $0.95(\mathrm{ddd}, 14.4,12.0,3.0)$ & \\
\hline 16 & $2.18(\mathrm{~m})$ & 35.1 & $5.22(\mathrm{dd}, 10.8,6.0)$ & 64.5 \\
\hline \multirow[t]{2}{*}{17} & 1.63 (overlapped) & 23.3 & $2.44(\mathrm{dd}, 13.2,6.0)$ & 49.5 \\
\hline & $0.80(\mathrm{dd}, 13.0,2.0)$ & & $1.88(\mathrm{dd}, 13.2,10.8)$ & \\
\hline 18 & $0.71(3 \mathrm{H}, \mathrm{t}, 7.5)$ & 7.0 & $0.89(3 \mathrm{H}, \mathrm{t}, 7.6)$ & 8.7 \\
\hline \multirow[t]{2}{*}{19} & 1.66 (overlapped) & 30.8 & 1.81 (overlapped) & 32.0 \\
\hline & $1.11(\mathrm{~m})$ & & 1.74 (overlapped) & \\
\hline 20 & & 36.9 & & 44.1 \\
\hline 21 & $2.41(\mathrm{~s})$ & 70.4 & & 175.8 \\
\hline \multirow[t]{2}{*}{22} & $3.91(\mathrm{dd}, 11.0,11.0)$ & 67.8 & & \\
\hline & $3.48(\mathrm{dd}, 11.0,3.5)$ & & & \\
\hline \multirow[t]{2}{*}{23} & $5.08(\mathrm{~d}, 11.0)$ & 72.6 & & \\
\hline & $4.87(\mathrm{~d}, 11.0)$ & & & \\
\hline $\mathrm{OH}$ & & & 2.21 (br s) & \\
\hline
\end{tabular}

a) Measured at $500 \mathrm{MHz}$. b) Measured at $125 \mathrm{MHz}$. c) Measured at $600 \mathrm{MHz}$.

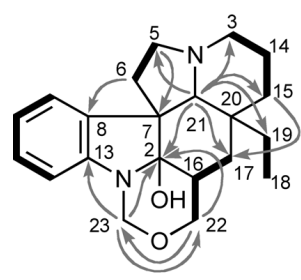

- ${ }^{1} \mathrm{H}-{ }^{1} \mathrm{H}$ COSY $\sim$ selected HMBC

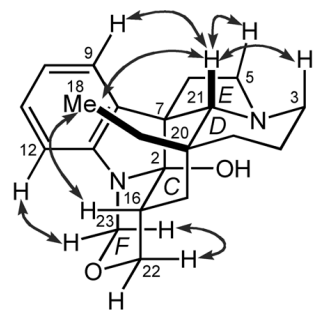

selected NOEs
Fig. 2. $\mathrm{HMBC},{ }^{1} \mathrm{H}-{ }^{1} \mathrm{H}$ COSY, and NOE Correlations of $\mathbf{1}$

group and extended its connection to $\mathrm{C}-17$ and $\mathrm{C}-2$, as suggested by the HMBC correlations from $\mathrm{H}-22$ to $\mathrm{C}-23$ and $\mathrm{C}-$ 2 as well as the ${ }^{1} \mathrm{H}-{ }^{1} \mathrm{H}$ COSY correlation of $\mathrm{H}_{2}-22 / \mathrm{H}-16 / \mathrm{H}_{2}-$ 17 , respectively. In addition, the $\mathrm{C}-20$ quaternary carbon with an ethyl side chain was linked to $\mathrm{C}-21, \mathrm{C}-17$, and $\mathrm{C}-15$, as shown by HMBC correlations of H-15/C-17, H-21/C-17, and $\mathrm{H}-21 / \mathrm{C}-19$. Other correlations in the HMBC spectrum were in complete accord with the proposed structure.

The relative configurations of compound $\mathbf{1}$ at the stereogenic centers were determined by analyzing ${ }^{1} \mathrm{H}-{ }^{1} \mathrm{H}$ coupling constants and nuclear Overhauser effect (NOE) correlations, as discussed below. As a result of the rigid character of the Aspidosperma skeleton, the relative configurations at C-7, C20, and $\mathrm{C}-21$ were restricted, i.e., $7 S^{*}, 20 R^{*}$, and $21 R^{*}$,
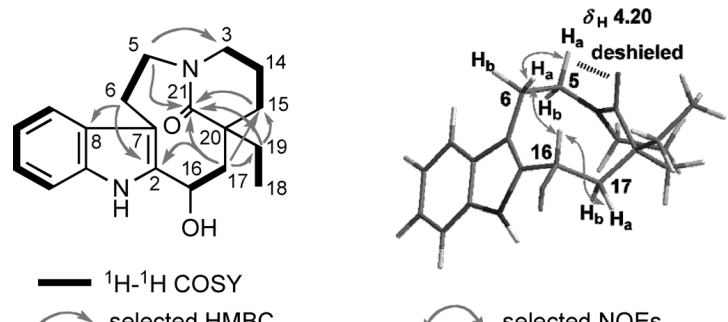

$\Upsilon$ selected NOEs

Fig. 3. $\mathrm{HMBC},{ }^{1} \mathrm{H}-{ }^{1} \mathrm{H}$ COSY, and NOE Correlations of 2

which were supported by the NOE correlations between $\mathrm{H}$ 21 and $\mathrm{H}-9$ and between $\mathrm{H}-21$ and $\mathrm{H}_{3}-18$ (Fig. 2). Furthermore, NOE observed between $\mathrm{H}_{3}-18$ and the angular methine proton on $\mathrm{C}-16$ and a large coupling constant $(J=11.0 \mathrm{~Hz})$ between this proton ( $\mathrm{H}-16)$ and $\mathrm{H} \alpha-22$ indicated the trans fusion of the $C / F$ ring. Therefore, alkaloid 1 possesses a novel hexacyclic skeleton incorporating a 1,3-oxazinane ring with relative configurations of $2 S^{*}, 7 S^{*}, 16 R^{*}, 20 R^{*}$, and $21 R^{*}$.

Compound 2, named kopsiyunnanine $\mathrm{H}$, was obtained as a light yellow amorphous solid. Its molecular formula was established as $\mathrm{C}_{19} \mathrm{H}_{24} \mathrm{~N}_{2} \mathrm{O}_{2}$ from HR-EI-MS $(\mathrm{m} / \mathrm{z} 312.1838$ $\left.\left[\mathrm{M}^{+}\right]\right)$. The UV and ${ }^{1} \mathrm{H}-\mathrm{NMR}$ spectra showed a typical indole chromophore. ${ }^{19)}$ The ${ }^{1} \mathrm{H}$ - and ${ }^{13} \mathrm{C}-\mathrm{NMR}$ data (Table 1 ) revealed the presence of an $\alpha, \beta$-disubstituted indole moiety, an amide function $\left(\delta_{\mathrm{C}} 175.8\right)$, an oxymethine $\left(\delta_{\mathrm{H}} 5.22, \delta_{\mathrm{C}}\right.$ $64.5)$, and an ethyl side chain $\left[\delta_{\mathrm{H}} 0.89(\mathrm{t}, J=7.6 \mathrm{~Hz}), \delta_{\mathrm{C}} 8.7\right]$.

Further 2D NMR experiments were carried out to connect the above partial fragments. As the HMBC correlations of $\mathrm{H}-$ 19/C-15, H-19/C-21, and H-17/C-19 suggested, the ethyl side chain was attached to $\mathrm{C}-15, \mathrm{C}-17$, and $\mathrm{C}-21$ via $\mathrm{C}-20$ (Fig. $3)$. Fragments $C(5)-C(6)$ and $C(3)-C(14)-C(15)$ revealed by ${ }^{1} \mathrm{H}-{ }^{1} \mathrm{H}$ COSY correlations were both linked to $\mathrm{C}-21$ of the amide group. HMBC correlations from $\mathrm{H}-5$ to $\mathrm{C}-3$ and $\mathrm{C}-21$, and from $\mathrm{H}-15$ to $\mathrm{C}-21$ supported the above linkages. On the other hand, fragment $\mathrm{C}(5)-\mathrm{C}(6)$ was attached to the indole moiety, as indicated by the HMBC correlations from $\mathrm{H}-6$ to C-8 and C-2. Fragment C(16)-C(17) was also attached to the indole moiety, based on the HMBC correlation observed from $\mathrm{H}-17$ to $\mathrm{C}-2$. C-16 of the $s p^{3}$ methine group gave a downfield chemical shift of $\delta_{\mathrm{C}} 64.5$, suggesting one hydroxyl group substitution. Thus, the structure of new compound 2 was elucidated as shown.

The relative configuration of the hydroxyl group on C-16 was established by analyzing the coupling constants and the NOE correlations (Fig. 3), as follows. One of the protons on C-5 showed a characteristic downfield chemical shift $\left(\delta_{\mathrm{H}}\right.$ 4.20), strongly deshielded by the anisotropy effect of the amide carbonyl group. Observation of the NOE correlation between that particular proton $(\mathrm{H}-5 \mathrm{a})$ and one of the protons on C-6 (H-6a: $\left.\delta_{\mathrm{H}} 3.48\right)$ indicated that they were on the same side of the ring. This was supported by the large coupling constant $(J=12.0 \mathrm{~Hz})$ between $\mathrm{H}-5 \mathrm{~b}$ and $\mathrm{H}-6 \mathrm{a}$, which was characteristic of an anti relationship (Fig. 4). On the other hand, the NOE correlation between H-6a and H-16, an oxymethine proton, was clearly observed, the latter of which possessed coupling constants $J=10.8 \mathrm{~Hz}$ and $6.0 \mathrm{~Hz}$ that were attributed to vicinal coupling with the two methylene protons on $\mathrm{C}-17$. The anti relationship of $\mathrm{H}-16$ and $\mathrm{H}-17 \mathrm{~b}$ $(J=10.8 \mathrm{~Hz})$ was proved by the NOE correlation between $\mathrm{H}-$ 16 and $\mathrm{H}-17 \mathrm{a}(J=6.0 \mathrm{~Hz})$. Although compound 2 had a con- 


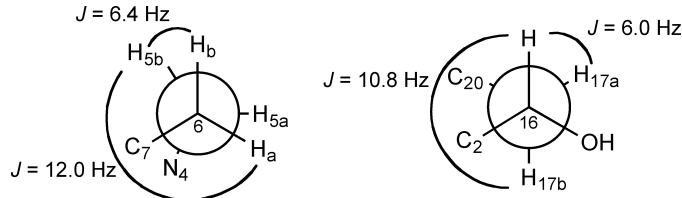

Fig. 4. Analysis of Coupling Constant of $\mathbf{2}$

formationally flexible nine-membered ring, taking together all the data mentioned above, the hydroxyl group on C-16 might have an alpha orientation. The positive Cotton effect at approximately $\lambda_{\max } 230 \mathrm{~nm}$ in the circular dichroism (CD) spectrum could be used to deduce the $R$ absolute configuration at C-20 in compound $2,{ }^{20)}$ leading to the $S$ configuration at $\mathrm{C}-16$.

\section{Experimental}

General Experimental Procedures Optical rotations were measured with a JASCO P-1020 polarimeter. CD was recorded on a JASCO J-720WI spectrometer. UV spectra were measured with a JASCO V-560 spectrophotometer. NMR spectroscopic data were recorded on a JNM A-500 or a JNM ECP-600 spectrometer, where $J$ values are given in Hz. EI-MS and HR-EIMS were recorded on a JEOL JMS GC-mate spectrometer with direct probe insertion at $70 \mathrm{eV}$. TLC was done on precoated silica gel 60 F254 plates (Merck, $0.25 \mathrm{~mm}$ thick) or precoated amino-silica gel plates (Fuji Silysia Chemical Ltd.). Column chromatography was carried out over silica gel 60 (Merck, 70-230 mesh) or amino-silica gel (Fuji Silysia Chemical Ltd., Chromatorex NH 100-200 mesh).

Plant Material Kopsia arborea Blume (Kopsia officinalis Tsiang et $\mathrm{P}$. T. LI) was collected from Xishuangbanna, Yunnan Province, China and identified by one of the authors, Professor Dr. Rongping Zhang. A voucher specimen (no. 20060401) was deposited at the Faculty of Pharmaceutical Sciences, Kunming Medical University.

Extraction and Isolation Extraction of the aerial part of $K$. arborea Blume (K. officinalis Tsiang et P. T. LI) $(9.0 \mathrm{~kg}$, dry weight) was carried out in the usual manner as has been described in detail before. ${ }^{20)}$ The alkaloids were partitioned primarily by silica open column chromatography using gradient $\mathrm{MeOH} / \mathrm{CHCl}_{3}$ solvent system. Eburenine $(3,7.1 \mathrm{mg})$ was obtained from the fraction eluted with $10-20 \% \mathrm{MeOH} / \mathrm{CHCl}_{3}$. The fraction eluted with $30-40 \% \mathrm{MeOH} / \mathrm{CHCl}_{3}$ was subjected to rechromatography on silica gel and the fraction eluted with $1-2.5 \% \mathrm{MeOH} / \mathrm{CHCl}_{3}$ gave (+)-eburnamonine $(5.6 \mathrm{mg})$. Successive separation of the fraction eluted with $3 \%$ $\mathrm{MeOH} / \mathrm{CHCl}_{3}$ on a silica gel open column $(80 \% n$-hexane/EtOAc) gave kopsiyunnanine $\mathrm{G}(\mathbf{1}, 1.2 \mathrm{mg})$, (-)-eburnamine $(15.0 \mathrm{mg})$, (-)-methyleburnamine $(1.2 \mathrm{mg}),(-)$-ethyleburnamine $(1.9 \mathrm{mg}),(+)$-isoeburnamine $(4.3$ $\mathrm{mg}),(+)$-methylisoeburnamine $(2.3 \mathrm{mg}),(+)$-ethylisoeburnamine $(1.8 \mathrm{mg})$, and $(+)$-eburnamenine $(3.5 \mathrm{mg})$. On the other hand, separation of the fraction eluted with $4 \% \mathrm{MeOH} / \mathrm{CHCl}_{3}$ on an amino-silica gel open column ( $n$ hexane/EtOAc in gradient) gave kopsiyunnanine $\mathrm{H}(\mathbf{2}, 2.1 \mathrm{mg})$.

Kopsiyunnanine G (1): Colorless amorphous solid, $[\alpha]_{\mathrm{D}}^{25}-72.9(c=0.07$, $\mathrm{CHCl}_{3}$ ). EI-MS $m / z$ (\%): $340\left(\mathrm{M}^{+}\right), 80$ (100). HR-EI-MS m/z: 340.2157 $[\mathrm{M}]^{+}$(Calcd for $\left.\mathrm{C}_{21} \mathrm{H}_{28} \mathrm{~N}_{2} \mathrm{O}_{2}: 340.2151\right)$. UV $\lambda_{\max }(\mathrm{MeOH}) \mathrm{nm}(\log \varepsilon)$ : 291.5 (3.59), 246.0 (3.96), 208.0 (4.40). CD (c=0.36 mM, MeOH, $\left.25^{\circ} \mathrm{C}\right) \Delta \varepsilon$ $(\lambda \mathrm{nm}):-11.7(211),-1.8(233), 0(250),+0.5(262), 0(275),-2.8$ (297), 0 (320). ${ }^{1} \mathrm{H}$ - and ${ }^{13} \mathrm{C}-\mathrm{NMR}$ data are shown in Table 1.

Kopsiyunnanine H (2): Light yellow amorphous solid, $[\alpha]_{D}^{18}-98.6$ $\left(c=0.09, \mathrm{CHCl}_{3}\right)$. EI-MS $m / z: 312\left(\mathrm{M}^{+}\right)$. HR-EI-MS $m / z: 312.1838[\mathrm{M}]^{+}$ (Calcd for $\left.\mathrm{C}_{19} \mathrm{H}_{24} \mathrm{~N}_{2} \mathrm{O}_{2}: 312.1828\right)$. UV $\lambda_{\max }(\mathrm{MeOH}) \mathrm{nm}(\log \varepsilon): 292.5$ (3.73), 283.5 (3.80), 224.0 (4.44), 204.0 (4.33). CD ( $c=0.3 \mathrm{~mm}, \mathrm{MeOH}$, $\left.24^{\circ} \mathrm{C}\right) \Delta \varepsilon(\lambda \mathrm{nm}):-17.9(211), 0(222),+21.4(229), 0(243),-3.5(270)$, 0 (309). ${ }^{1} \mathrm{H}-$ and ${ }^{13} \mathrm{C}-\mathrm{NMR}$ data are shown in Table 1.

Eburenine (3): The detailed spectroscopic data of $\mathbf{3}$ have not been published so far and thus, we present them here. ${ }^{1} \mathrm{H}-\mathrm{NMR}\left(\mathrm{CDCl}_{3}, 500 \mathrm{MHz}\right) \delta$ : $7.51(1 \mathrm{H}, \mathrm{d}, J=7.5 \mathrm{~Hz}, \mathrm{H}-12), 7.33(1 \mathrm{H}, \mathrm{d}, J=7.5 \mathrm{~Hz}, \mathrm{H}-9), 7.29(1 \mathrm{H}$, ddd, $J=7.5,7.5,1.0 \mathrm{~Hz}, \mathrm{H}-11), 7.16(1 \mathrm{H}, \mathrm{ddd}, J=7.5,7.5,1.0 \mathrm{~Hz}, \mathrm{H}-10), 3.19$ $(1 \mathrm{H}$, overlapped, H-5a), $3.18(1 \mathrm{H}$, overlapped, H-3a), $3.11(1 \mathrm{H}$, ddd, $J=14.0$, $14.0,5.0 \mathrm{~Hz}, \mathrm{H}-16 \mathrm{a}), 2.76(1 \mathrm{H}, \mathrm{ddd}, J=14.0,10.0,3.5 \mathrm{~Hz}, \mathrm{H}-16 \mathrm{~b}), 2.60(1 \mathrm{H}$, m, H-5b), 2.47 (1H, ddd, $J=13.5,13.5,3.0 \mathrm{~Hz}, \mathrm{H}-17 \mathrm{a}), 2.41(1 \mathrm{H}, \mathrm{s}, \mathrm{H}-21)$,
2.18 (1H, overlapped, H-3b), 2.17 (1H, overlapped, H-6a), $1.86(1 \mathrm{H}, \mathrm{m}, \mathrm{H}-$ 14a), $1.65(1 \mathrm{H}, \mathrm{dd}, J=12.5,5.0 \mathrm{~Hz}, \mathrm{H}-6 \mathrm{~b}), 1.61(1 \mathrm{H}, \mathrm{m}, \mathrm{H}-17 \mathrm{~b}), 1.54(1 \mathrm{H}$, m, H-14b), $1.48(1 \mathrm{H}$, br d, $J=14.0 \mathrm{~Hz}, \mathrm{H}-15 \mathrm{a}), 1.01(1 \mathrm{H}, \mathrm{ddd}, J=13.5,13.5$, $5.0 \mathrm{~Hz}, \mathrm{H}-15 \mathrm{~b}), 0.64\left(2 \mathrm{H}, \mathrm{m}, \mathrm{H}_{2}-19\right), 0.50\left(3 \mathrm{H}, \mathrm{t}, J=7.5 \mathrm{~Hz}, \mathrm{H}_{3}-18\right) .{ }^{13} \mathrm{C}-$ NMR $\left(\mathrm{CDCl}_{3}, 125 \mathrm{MHz}\right) \delta$ : 192.4 (C-2), 154.5 (C-13), 147.1 (C-8), 127.4 (C-11), 125.1 (C-10), 121.0 (C-9), 120.1 (C-12), 78.9 (C-21), 61.2 (C-7), 54.5 (C-5), 52.0 (C-3), 36.5 (C-20), 35.1 (C-6), 33.2 (C-15), 29.7 (C-19), 27.2 (C-17), 23.7 (C-16), 22.0 (C-14), 7.2 (C-18). CD ( $c=0.37 \mathrm{~mm}, \mathrm{MeOH}$, $\left.25^{\circ} \mathrm{C}\right) \Delta \varepsilon(\lambda \mathrm{nm}):+10.8(203), 0(220),-3.2(226), 0(230),+1.0(237), 0$ (241), -0.3 (244), 0 (246), + 10.3 (270), 0 (382).

Acknowledgements This work was supported by a Grant-in-Aid for Scientific Research from the Ministry of Education, Culture, Sports, Science and Technology, Japan and The Uehara Memorial Foundation.

\section{References}

1) Middleton D., Harvard Pap. Bot., 9, 89-142 (2000).

2) Kam T. S., Lim K. H., "The Alkaloids,” Vol. 66, ed. by Cordell G. A., Academic Press, Amsterdam, 2008, pp. 1-105.

3) Subramaniam G., Hiraku O., Hayashi M., Koyano T., Komiyama K., Kam T. S., J. Nat. Prod., 70, 1783-1789 (2007).

4) Subramaniam G., Hiraku O., Hayashi M., Koyano T., Komiyama K., Kam T. S., J. Nat. Prod., 71, 53-57 (2008).

5) Subramaniam G., Kam T. S., Tetrahedron Lett., 48, 6677-6680 (2007).

6) Kam T. S., Lim K. H., Yoganathan K., Hayashi M., Komiyama K., Tetrahedron, 60, 10739-10745 (2004).

7) Sekiguchi M., Hirasawa Y., Zaima K., Hoe T. C., Chan K. L., Morita H., Heterocycles, 76, 867-874 (2008).

8) Sekiguchi M., Hirasawa Y., Zaima K., Hoe T. C., Chan K. L., Morita H., Heterocycles, 75, 2283-2288 (2008).

9) Jakob M., Cheryl A. C., Michael A. K., Org. Lett., 10, 1437-1440 (2008).

10) Hong X. C., France S., Mejía-Oneto J. M., Padwa A., Org. Lett., 8, $5141-5144$ (2006).

11) Lim K. H., Low Y. Y., Kam T. S., Tetrahedron Lett., 47, 5037-5039 (2006).

12) Kitajima M., J. Nat. Med., 61, 14-23 (2007)

13) Kitajima M., Tanaka A., Kogure N., Takayama H., Tetrahedron Lett., 49, 257-260 (2008).

14) Kogure N., Kobayashi H., Ishii N., Kitajima M., Wongseripipatana S., Takayama H., Tetrahedron Lett., 49, 3638-3642 (2008).

15) Kogure N., Nozoe A., Kitajima M., Takayama H., Heterocycles, 79, $1101-1105$ (2009).

16) Takayama H., Kitajima M., Kogure N., Current Org. Chem., 9, $1445-$ 1464 (2005).

17) Takayama H., Mori I., Kitajima M., Aimi N., Lajis N. H., Org Lett., 6, 2945-2948 (2004).

18) Yamada Y., Kitajima M., Kogure N., Takayama H., Tetrahedron Lett., 50, 3341-3344 (2009).

19) Wu Y. Q., Kitajima M., Kogure N., Zhang R. P., Takayama H., Tetrahedron Lett., 49, 5935-5938, 6596 (2008).

20) Wu Y. Q., Suehiro M., Kitajima M., Matsuzaki T., Hashimoto S., Nagaoka M., Zhang R. P., Takayama H., J. Nat. Prod., 72, 204-209 (2009).

21) Wu Y. Q., Kitajima M., Kogure N., Wang Y. S., Zhang R. P., Takayama H., J. Nat. Med., 63, 283-289 (2009).

22) Sattar E. A. A., Olemy M. M. E., Mossa J. S., Saudi Pharmaceut. J., 2, 135-139 (1994).

23) Feng X. Z., Kan C., Husson H. P., Potier P., Kan S. K., Lounasmaa M., J. Nat. Prod., 47, 117-122 (1984).

24) Goh S. H., Ali R. M., Wong W. H., Tetrahedron, 45, 7899-7920 (1989).

25) Kam T. S., Tan P. S., Wei C., Phytochemistry, 33, 921—924 (1993).

26) Vercauteren J., Kerharo J., Morfaux A.M., Massiot G., Men-Olivier L. L., Men J. L., Phytochemistry, 19, 1959-1961 (1980).

27) Atta-ur-Rahman, Zaman K., Perveen S., Habib-ur-Rehman, Muzaffar A., Choudhary M. I., Pervin A., Phytochemistry, 30, 1285-1293 (1991).

28) Kam T. S., Tan P. S., Chuan C. H., Phytochemistry, 31, 2936-2938 (1992). 